\title{
A network-based analysis of key pharmacological pathways of Corilagin acting on Breast cancer and experimental validation
}

\author{
Xue Zhong ${ }^{\#, 1}$, Jintian Tang ${ }^{2}$, Wenyue Long ${ }^{1}$, Xu Cai ${ }^{2}$, Huiguo Wang ${ }^{*}, 1$, and Qian Li ${ }^{*}, 2$ \\ ${ }^{1}$ College of Life Science and Technology, Dalian University, Liaoning 116622, China \\ ${ }^{2}$ Department of Engineering Physics, Institute of Medical physics, Tsinghua University, Beijing 100084, China
}

\begin{abstract}
The combination of network pharmacological methods and experimental verification has revealed the underlying molecular mechanism of Corilagin in the treatment of breast cancer. In this study, we used Swiss Target Prediction database network to predict the possible candidate targets and pathways of Coilagin, and collect breast cancer targets and pathways of CooLGeN. The result of network pharmacological screen indicated that Bcl is a key pathway for Corilagin in the treatment of breast cancer. The CCK-8 experiment also proved that Corilagin could inhibit the proliferation of MCF-7 cells. Western Blot was used for the validation of network pharmacological results, which showed that Corilagin could increase the apoptosis rates (Bcl-2/Bax) of MCF-7 cells, and up-regulates the apoptotic protein Caspase-3 and changes the Bcl-2 family proteins expression in MCF-7 cells. All results suggest that Corilagin has the potential therapeutic effects on breast cancer, and may be useful for adjuvant chemotherapy.
\end{abstract}

\section{Introduction}

The increasing cancer incidence is a heavy burden to the world [1]. Compared to other cancers, the morbidity of breast cancer is the highest. Because of the limitations of early diagnosis and treatment. In developing countries, breast cancer has become the second leading cause of death for women [2-4]. Breast cancer cases accounts for $25 \%$ of all new cancer cases, and $13.7 \%$ of all cancer cases [5]. Although there are many options for treating Breast cancer, such as surgery, chemotherapy, biological immunotherapy, radiotherapy and targeted molecular therapy [6,7], the recovery of breast cancer is not as good as expected. In addition, there are more than 410,000 women die from breast cancer each year breast for resistant current therapies [6]. Looking for drugs with high efficiency and low side effects is the goal of antibreast cancer drug research. The development of bioactive compounds from natural sources to treat breast cancer has gradually become a focus of attention.

Corilagin is a tannin component isolated from a variety of Chinese herbal medicines or medical plants such as Phyllanthus Emblica L, Dimocarpus Longan Lour [8], Ricinodendron heudelotii leaves [9], Phyllanthus niruri [10] and P. amarus [11]. Corilagin, $\mathrm{C}_{27} \mathrm{H}_{22} \mathrm{O}_{18}$, molecular weight 634.45. Many studies showed that Corilagin has many biological functions, and the most significant is anti-tumor activity [12-14]. The chemical structure of drug affects the protein target, which is thought-provoking and can clearly show the pharmacological effects of Corilagin. Significantly, network pharmacology is based on systems biology, computer technology, gene redundancy, and multiple pharmacological theories. It is an effective tool to elucidate the complex interactions between drugs and biological systems. Previously, our group has evaluated the anti-tumor effects of Corilagin in MCF-7, A549, SW480, and Hela cells. The results showed that the MCF-7 cell is the most sensitive cell to the cytotoxic effects of Corilagin than other cancer cells. The MCF-7 cell line is the most studied human breast cancer cell line in the world. The study of this cell line has greatly affected the cure of breast cancer and the prognosis of cancer patients. Our study found that MCF-7 cells are sensitive to Corilagin, and its resistance to breast cancer has not been confirmed in the past literature. This study used the MCF-7 cell line to investigate the inhibitory effect of Corilagin on breast cancer and applied molecular techniques to expound the possible underlying mechanisms.

\section{Methods}

\subsection{Chemicals}

Corilagin (purity $\geq 98 \%$, HPLC) was obtained from Solarbio (Beijing, China). DMEM, FBS and $0.25 \%$ trypsin-EDTA solution (Gibco BRL, Carlsbad, CA, USA); Penicillin-streptomycin solution (Logan, UT, USA); Cell Counting Kit-8 (CCK-8, Dojindo, Japan); Chemiluminescent (ELC) kit (Beyotime, China); BCA Protein Assay Kit (Beyotime, China).

\footnotetext{
* Corresponding author: Huiguo Wang, E-mail: wanghuiguo163@163.com
} 


\subsection{Screening for the potential therapeutic targets of Corilagin and functional analysis}

For finding possible target, we input Corilagin's threedimensional (3D) structure into STITCH (http://stitch.embl.de/) [15] and Swiss Target Prediction (http: //www.swisstargetprediction.ch/) [16]. Then we performed the functional analysis of the obtained target protein, and visualized and integrated discovery research on functional annotations of key targets via DAVID (http://david.abcc.ncifcrf.gov/).

\subsection{Screening for the potential therapeutic targets of Breast cancer and network Construction}

Online text mining server CooLGeN is used to search for relevant targets for breast cancer (http://ci.smu.edu.cn/CooLGeN/). The parameter settings were as follows. The keyword search was "breast cancer", the impact factor was set to $4 \sim 50$, the year was $1989 \sim 2019$, the species was selected "all human genes". The relevant targets obtained were compared with Corilagin related targets. Venn Diagram (http://bioinformatics.psb.ugent.be/webtools/Venn/) was used to find common goals. Protein-protein interaction (PPI) in the human genome is extracted from STRING version 9.05 (https://string-db.org/cgi/input.pl). Database Visualization and integrated discovery software was used for functional analysis (DAVID, http://david.abcc.ncifcrf.gov/home.) The pathways came from KEGG (http://www.genome.jp/kegg/). The common-target network of Corilagin and disease-target network of Breast cancer was constructed by Cytoscape v3.7.0 software which is a useful tool for analysis and visualization of the biological network.

\subsection{Cell viability assay}

MCF-7 cells $\left(6 \times 10^{3}\right.$ cells/well $)$ were separated in 96well plates and cultured with $0,20,40,60,80,100$, $120,140,160,180$ and $200 \mu \mathrm{M}$ of Corilagin. After 24 $\mathrm{h}$ and $48 \mathrm{~h}$ treatments, cells were subjected to 10 $\mu \mathrm{l} /$ well of CCK-8 solution and incubated for $3 \mathrm{~h}$ at $37^{\circ} \mathrm{C}$ with $5 \% \quad \mathrm{CO}_{2}$. Varioskan Flash (Thermo Scientific, USA) was used to measure the optical density of each well at $450 \mathrm{~nm}$.

\subsection{Western blot assay}

MCF-7 cells were exposed to Corilagin (0, 40, 80 and $120 \mu \mathrm{M})$ for $48 \mathrm{~h}$. Cells were lysed in RIPA lysis buffer and the protein concentration was determined by the BCA protein assay kit. Primary antibodies against Bax, Caspase-3, Bcl-2, Bcl-xL, and $\beta$-Actin were sequentially incubated on the membrane, and the membrane was then conjugated with a horseradish peroxidase (HRP) secondary antibody incubate. Protein bands were visualized and detected using enhanced chemiluminescence (ELC) kits and Image
Quant LAS 4000s (GE, USA).

\subsection{Statistical analysis of data}

The data were analyzed by using SPSS 21.0 and GraphPad Prism 8.0, and were expressed as $\bar{x} \pm s$.

\section{Results}

\subsection{The candidate targets of Corilagin}

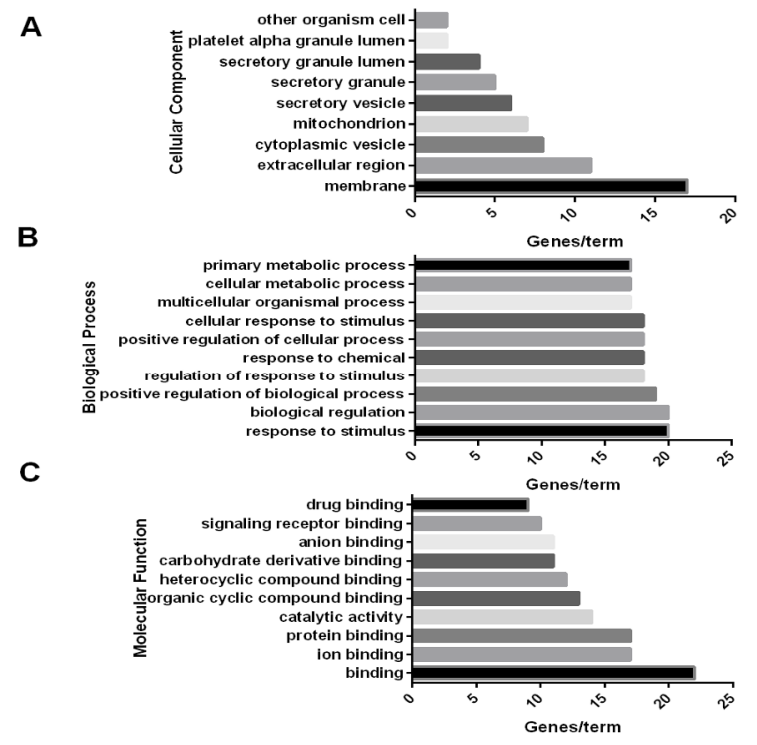

Fig. 1. (A) Cellular component (B) Biological process (C) Molecular function $(P<0.05)$

First, two database software were used to identify Corilagin's targets including 100 target proteins and Gene ID. In order to found their characteristics at the molecular level and pathway mechanism, we performed functional analysis of these targets through DAVID. Therefore, the top 10 results of Cellular Component, Molecular Function, Biological Process were shown in Fig.1(A-C). The largest number of genes in Cell component is the "membrane". The largest number of genes in Biological process is the "response to stimulus". The largest number of genes in Molecular function is the "binding". Corilagin is associated with cancer.

\subsection{KEGG and Common-target network}

In Fig.2(A), the result showed that there were totally 57 pathways (Qvalue < 0.05) affected by the active ingredients of Corilagin, including "neuroactive ligandreceptor interaction, morphine addiction, vascular smooth muscle contraction, pathways in cancer, proteoglycans in cancer, PI3K-Akt signaling pathway, rap1 signaling pathway, MAPK signaling pathway, ras signaling pathway, chemokine signaling pathway". The CoolGeN online analysis tool was applied to searches for 473 genes with the key word "breast cancer". Compared with 57 targets of Corilagin, 22 common targets were 
obtained, and showed in Fig.2(B), MMP2, PTPN1, MET, AXL, ALK,

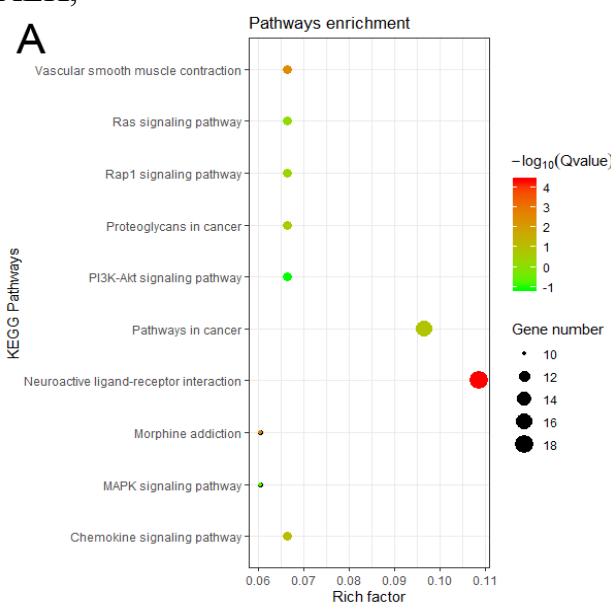

B

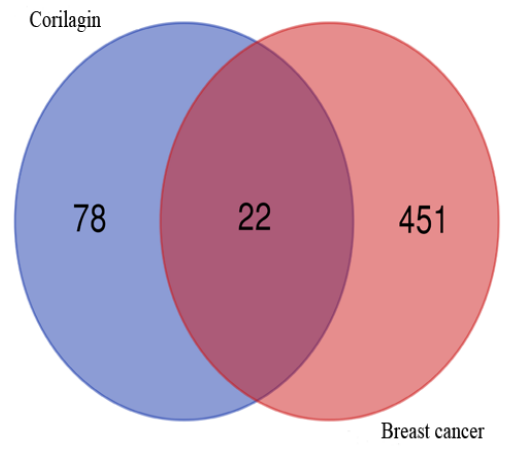

Fig. 2. (A) KEGG enrichment analysis. (B) The commontarget network of Corilagin and Breast cancer $(P<0.05)$.

LGALS3, SHBG, FGF2, IGF1R, BCL2L1, TERT, ABCB1, SERPINE1, STS, TP53, HPSE, PTGS1, VEGFA, ESR2, PRKCA, HSP90AA1, VDR, which may be the main target of Corilagin in treating breast cancer.

\subsection{PPI Network}

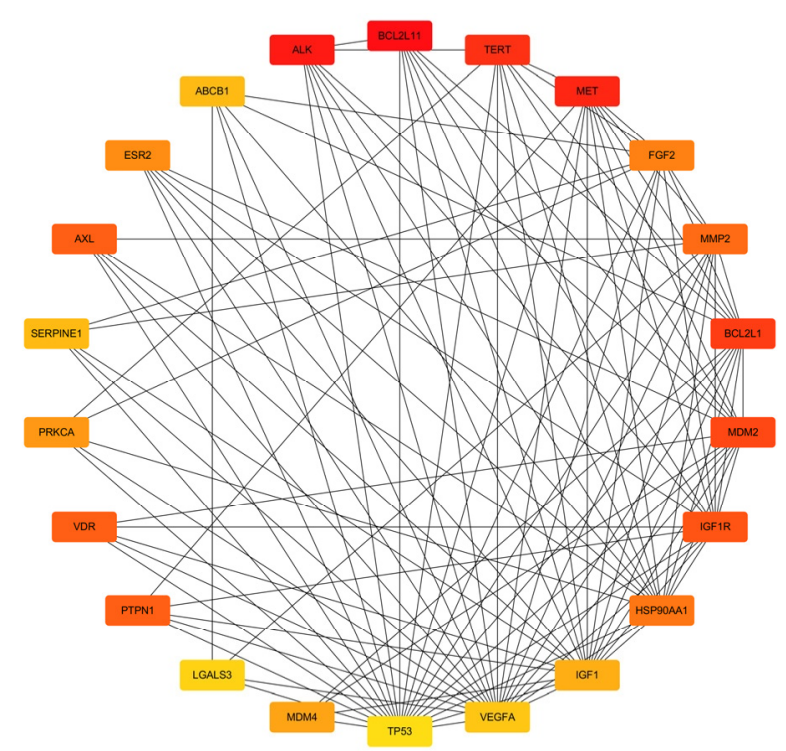

Fig. 3 Common-target networks of Corilagin and Breast cancer.
As shown in Fig.3, the PPI network was composed of 22 common targets with 22 nodes and 77 edges. The average node degree is 7 . (P-value $<1.0 \mathrm{e}-16)$. Nodes with large degree values are the key targets in PPI. It is worth noting that the BCL2L1 protein (BCL2L1, degree $=13$ ) was the most important protein in the diseasetarget network, and plays an important role in the pathogenesis of Breast cancer. In addition, MET, IGF1, AKL, IGF1R, and MDM2 are also considered important Breast cancer targets based on degree and betweenness centrality.

\subsection{Cell viability analysis}

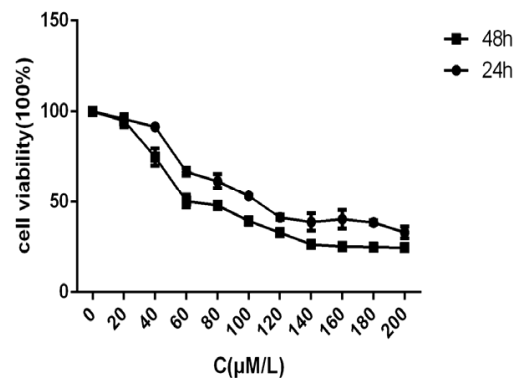

Fig. 4. Cell viability of MCF-7 cells was examined by CCK-8 assay after treatment with different concentrations of Corilagin. $(P<0.05)$

As the CCK-8 assay results showed in Fig.4, Corilagin can reduce the viability of MCF-7 cells depending on the does and time. The $\mathrm{IC}_{50}$ value of Corilagin was 95.86 $\mu \mathrm{M}$ for $48 \mathrm{~h}$. As the concentration of Corilagin showed significantly decrease from $40 \mu \mathrm{M}$ to $120 \mu \mathrm{M}$, we used this dose range to treat MCF-7 cells for the subsequent experiments.

\subsection{Western blot analysis}
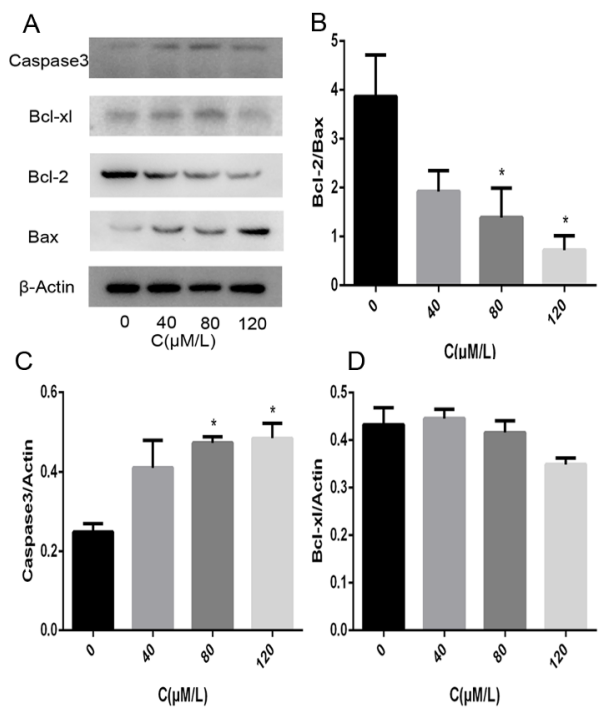

Fig. 5. (A-D) Western blot analysis of Bcl-2, Bax, Caspase-3, $\mathrm{Bcl}-\mathrm{xL}$, proteins in Corilagin treated $\mathrm{MCF}-7$ cells with various concentrations. $\beta$-Actin was used as a control. $(P<0.05)$ 
To clarify the Corilagin inhibits the growth of MCF-7 cells, we tested the key protein signaling pathways of network pharmacological screening by Western blot experiments. Accordingly, the expression of other regulators (Bax, Caspase-3, Bcl-2, Bcl-xL) in the apoptotic pathways were examined after cultured with Corilagin for $48 \mathrm{~h}$. Compared with the control group, the expression of Bax and Caspase- 3 was increased significantly, and the levels of Bcl-2 and $\mathrm{Bcl}-\mathrm{xL}$ were reduced $(P<0.05)$ (Fig. 5A-D). All data indicated that Corilagin induced apoptosis in MCF-7 cells.

\section{Conclusions}

A network pharmacological approach was developed to reveal the underlying molecular mechanism of Corilagin in the treatment of breast cancer. Network analysis is successfully predicted that Corilagin responds to breast cancer by targeting 22 candidate targets and 10 pathways (included cell proliferation, migration, and apoptosis). Network pharmacological screening found that $\mathrm{Bcl}$ is a key protein for Corilagin in the treatment of breast cancer. Corilagin has been experimentally verified to inhibit the proliferation of MCF-7 cells. Meanwhile, the decrease of Bcl-2/Bax and the decrease in Bcl-xl lead to an increase in Caspase-3 expression, and eventually induce apoptosis in MCF-7 cells. Bcl-2 family is an essential regulatory protein in the process of cell apoptosis. Bcl-2 inhibits cell apoptosis by inhibiting the release of cytochrome $\mathrm{c}$ and other mediating apoptosis factors from mitochondria to the cytoplasm. Subsequent experiments should further prove the effect of Corilagin on the apoptosis rate of MCF-7 cells and the verification of other pathways. In conclusion, due to the complexity and pathogenesis of tumor targets, it is very feasible to conduct further research based on the results direction of network pharmacology. Corilagin can be used as a new candidate anti-tumor drug for treating breast cancer patients.

\section{Acknowledgements}

We also would like to thank the members of the Department of Medical Physics at Tsinghua University for their helpful discussions. This research did not receive any specific grant from funding agencies in the public, commercial, or not-for-profit sectors.

\section{References}

1. X. Liu, F. Liu, S. Zhao, et al., Carbohydr. Polym. 215 99-107 (2019).

2. Nejati-Azar. A, Alivand. MR, Per. Med. 15 279-289 (2018).

3. Bayat. S, Mansoori Derakhshan. S, Mansoori Derakhshan. N, et al., J. Cell. Biochem. 120 91729180 (2019) .

4. Z. Luo, X. Hu, H. Xiong, et al., Carbohydr Polym. 151 1027-1033 (2016).
5. S. Vijayarathna, S. Sasidharan, Asian. Pac. J. Trop. Biomed. 2 826-9 (2012).

6. MS. Chambers, HS. Rugo, JK. Litton, et al., J. sAm. Dent. Assoc. 149 291-298 (2018).

7. M. Ariana, N. Arabi, M. Pornour, et al., J. Cell. Biochem. 119 2168-2178 (2018).

8. YY. Tang, XM. He, J. Sun, et al., Molecules. 24 (2019).

9. OF. Yakubu, AH. Adebayo, EEJ. Iweala, et al., Heliyon. 5 (2019).

10. MFS. Silva, LMA. Silva, AL. Quintela, et al., J. Chromatogr. B. Analyt. Technol. Biomed. Life. Sci. 1120 51-61 (2019).

11. A. Gupta, AK. Singh, R. Kumar, et al., Molecules. 24 (2019).

12. N. Aziz, A. Rasul, SA. Malik, et al., Pak. J. Pharm. Sci. 32 785-792 (2019).

13. DO. Kennedy, EL. Wightman, Adv. Nutr. 2 32-50 (2011).

14. WT. Yang, GH. Li, ZY. Li, et al., Evid Based Complement Alternat Med. 2016 (2016).

15. M. Kuhn, C. von Mering, M. Campillos, LJ. Jensen, P. Bork, Nucleic. Acids. Res. 36 (2007).

16. D. Gfeller, A. Grosdidier, M. Wirth, et al., Nucleic. Acids. Res. 42 (2014). 